\title{
Design of Ultrasonic Transducer for Secondary Wave Generations with High Directivity
}

\author{
Chol-Hak Kim, Myong-Jin Kim and Chol-Su Ri \\ Department of Physics, Kim Il Sung University, Pyongyang, Democratic People's Republic of Korea.
}

\begin{abstract}
(Received 30 November 2017; accepted 11 April 2018)
In this paper, we described a method of designing ultrasonic transducer which simultaneously radiates two finiteamplitude ultrasonic waves to produce the secondary waves with high directivity. For nonlinear effects, it is necessary that the frequencies of two primary waves are coincident with natural frequencies of the ultrasonic transducer. The main problem here is to predict the resonance frequencies of the first mode as well as higher modes. While the first resonance frequency of the transducer can be estimated easily, it is not trivial to do higher ones. When the length of transducer is much greater than its diameter, this problem is reduced to one-dimensional and higher mode frequencies are nothing but multiples of the first mode frequency. However, such a case is seldom encountered. Using the transfer matrix method, we obtained the resonance frequencies of the transducer analytically and compared these with numerical results from the simulation. The theoretical and simulation results are in good agreement with the difference of $3-6 \mathrm{kHz}$.
\end{abstract}

\section{INTRODUCTION}

Generally, polaroid-type sensors of Murata and SensComp have a directivity of about $20^{\circ}$ at working frequencies, meaning that the beam is about $35 \mathrm{~cm}$ wide at a distance of one meter from the sound source. ${ }^{1-3}$ Such a directivity limits application in several areas, such as detection and ranging in underwater and air, non-destructive testing of solid materials and medical inspection, etc. One possible way to improve directivity while keeping the frequencies the same is the use of nonlinear effects. The nonlinear effects in the propagation of sound waves have been discussed by many researchers. ${ }^{4-10}$

When two finite-amplitude primary waves propagate together in fluids, the sum and difference frequency waves as well as higher harmonics are produced as secondary waves, owing to the nonlinear interaction between the primary waves. From the absorptive properties of the medium, which prefer higher frequencies to lower ones, there remains only the difference frequency wave in the far-field. ${ }^{11-14}$ Darvennes and Hamilton showed that the directivity of the difference frequency wave in the far-field is governed by the product of the primary directivities, which becomes sharper. This observation, commonly known as the product directivity (PD) model has been widely used for many acoustic applications, especially for parametric array loudspeakers. ${ }^{15,16}$ The parametric loudspeaker was first devised by Yoneyama et al., who showed that an audio signal with a high directivity could be produced from ultrasonic waves due to the nonlinearity of the air. ${ }^{17}$ Since then, many later works have been done for the digital beam steering for parametric loudspeakers including the digital implementation of a beam steering algorithm, active noise control system, and the introduction of pre-processing methods. ${ }^{18-21}$

In spite of these successes of the PD model, there are some mismatches between the measured directivities and the theoretical results. Shi and Gan revisited the original theories, proposed three modified models for the directivity of the dif- ference frequency waves, and compared the numerical results with experimental results. ${ }^{22}$ According to their results, the shorter the distance between the sources of primary waves, the higher the directivity of the difference frequency wave. It is, therefore, obvious that a higher directivity of difference frequency wave can be obtained if two primary waves are radiated by one transducer. For doing so, the resonance frequencies of a transducer have to be separated as far as one of interest and the transducer must be designed so as to satisfy such a condition. It is easy to estimate the first mode frequency, but it is difficult to do higher modes. If a transducer satisfies the condition such that the length should be much greater than the diameter, higher mode frequencies become multiples of the first mode, but in other cases the evaluation is not so simple. In this paper, the resonance frequencies of the transducer are theoretically found using the transfer matrix method, and its validity is confirmed through the comparison with the simulation results using ANSYS 14.5.

\section{DESIGN PROCEDURE}

The transfer matrix expresses the relationship between the forces and velocities at both end-sides of the medium through which a plane wave propagates. The transfer matrix method has been applied for designing some acoustic structures, especially layered structures and simulating or analysing the propagation of sound waves within such structures. ${ }^{23,24}$

For the constant cross section, the transfer matrix is given as follows: ${ }^{25}$

$$
\left(\begin{array}{l}
\dot{\xi}_{2} \\
\dot{F}_{2}
\end{array}\right)=\left(\begin{array}{cc}
\cos k l & \frac{-i \sin k l}{\rho c s} \\
-\rho c s \sin k l & \cos k l
\end{array}\right)\left(\begin{array}{l}
\dot{\xi}_{1} \\
\dot{F}_{1}
\end{array}\right) ;
$$

where $\xi_{1}$ and $F_{1}$ are the vibration velocity of the medium particle and the force exerted on the incident boundary, respectively, and $\xi_{2}$ and $F_{2}$ are the counterparts on the opposite one. 


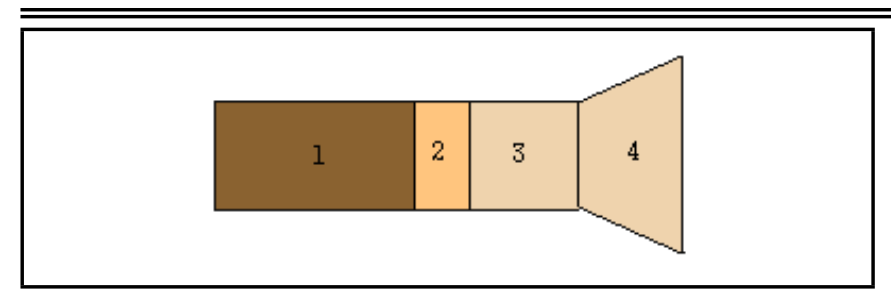

Figure 1. Transducer's shape: 1-steel, 2-PZT4, 3 and 4-aluminium.

$\rho$ denotes the density of the medium, $c$ is the sound velocity, $s$ is the cross section, $k$ is the wave number, and $l$ is the length of the medium. For a given nodal position of an ultrasonic transducer, one can construct the transfer matrices in both parts of the node point and apply these matrices and boundary conditions to obtain the resonance frequencies.

Now, let us apply the transfer matrix method to design the transducer which does not satisfy the one-dimensional condition (i.e., the condition that the length should be much greater than the diameter). Fig. 1 shows the shape of the transducer, which consists of four parts with three kinds of materials.

The node point is on the boundary side between the sector 1 and 2 of the transducer.

For the longitudinal mode of the transducer, the transfer matrix on the right part of the node point is:

$$
\begin{array}{r}
\left(\begin{array}{c}
\dot{\xi}_{2} \\
F_{2}
\end{array}\right)=\left(\begin{array}{cc}
\frac{a \sin k_{3} l_{3}}{k_{3}}+\frac{\cos k_{3} l_{3}}{\left(1+a l_{3}\right)} & \frac{-i \sin k_{3} l_{3}}{\rho_{3} c_{3} s_{2}\left(1+a l_{3}\right)} \\
B & D
\end{array}\right) \\
\left.\begin{array}{cc}
\cos k_{2} l_{2} & \frac{-i \sin k_{2} l_{2}}{\rho_{2} c_{2} s_{2}} \\
\cos k_{2} l_{2}
\end{array}\right) \\
-\left(\begin{array}{cc}
\cos c_{2} s_{2} \sin k_{2} l_{2} & \begin{array}{c}
\cos k_{1} l_{1} l_{1} \\
\rho_{1} c_{1} s_{1}
\end{array} \\
-\rho_{1} c_{1} s_{1} \sin k_{1} l_{1} & \cos k_{1} l_{1}
\end{array}\right)\left(\begin{array}{l}
\dot{\xi}_{0} \\
F_{0}
\end{array}\right) ; \\
B=\frac{\rho_{3} c_{3} s_{2} a^{2} \sin k_{3} l_{3}}{i k_{3}^{2}-\frac{\rho_{3} c_{3} s_{2} a \sin k_{3} l_{3}\left(1+a l_{3}\right)}{i k_{3}}} \\
-i \rho_{3} c_{3} s_{2} \sin k_{3} l_{3}\left(1+a l_{3}\right)+\frac{\rho_{3} c_{3} s_{2} a \cos k_{3} l_{3}}{i k_{3}} \\
-i \rho_{3} c_{3} s_{2} \sin k_{3} l_{3}\left(1+a l_{3}\right)+\frac{\rho_{3} c_{3} s_{2} a \cos k_{3} l_{3}}{i k_{3}} ; \\
D=\cos k_{3} l_{3}\left(1+a l_{3}\right)-\frac{a \sin k_{3} l_{3}}{k_{3}} ;
\end{array}
$$

where $F_{0}$ and $\dot{\xi}_{0}$ are the force and vibration velocity on the node point, respectively, and $F_{2}$ and $\dot{\xi}_{2}$ are the ones on the radiating surface. Eq. (2) could be rewritten simply as follows:

$$
\left(\begin{array}{l}
\dot{\xi}_{2} \\
F_{2}
\end{array}\right)=\left(\begin{array}{ll}
a_{11} & a_{12} \\
a_{21} & a_{22}
\end{array}\right)\left(\begin{array}{l}
\dot{\xi}_{0} \\
F_{0}
\end{array}\right) .
$$

Taking into account that the vibrational velocity is zero on the node point and the force is also zero on the radiating surface, we obtain:

$$
a_{22}=0 .
$$

We can determine the length of the right part of node point of the transducer with the natural frequency of $20 \mathrm{kHz}$ using Eq. (4).

Applying the same procedure for the left part, the length can be determined so as to give the natural frequency of $20 \mathrm{kHz}$.
Table 1. Materials and geometric dimensions of the ultrasonic transducer.

\begin{tabular}{|c|c|c|c|c|}
\hline$N_{\mathrm{o}}$ & 1 & 2 & 3 & 4 \\
\hline Material & Steel & PZT-4 & aluminium & aluminium \\
\hline Length $(\mathrm{mm})$ & 65.7 & 10 & 19 & 25 \\
\hline Diameter $(\mathrm{mm})$ & 30 & 30 & 30 & 40 \\
\hline Density $\left(\mathrm{kg} / \mathrm{m}^{3}\right)$ & 7800 & 7500 & 2690 & 2690 \\
\hline
\end{tabular}

Table 1 shows the calculated size and used material of every sector for the transducer with the natural frequency of $20 \mathrm{kHz}$.

For the fixed lengths of each part of the transducer, we can write an expression in terms of the resonance frequencies using Eq. (4). From Table 1, Eq. (4) transforms into the following:

$$
\begin{gathered}
-0.00002 i \sin (0.000013 f)[\cos (0.00006 f) \\
\cdot\left(-\frac{\left(1.8252 \cdot 10^{8} i\right) \cos (0.00005 f)}{f}\right. \\
-26464.78 i \sin (0.00005 f)-\frac{1.88 \cdot 10^{12} i \sin (0.00005 f)}{f^{2}} \\
\left.+\frac{2.74 \cdot 10^{8} i \sin (0.00005 f)}{f}-17643.18 i\right) \\
\cdot\left(1.5 \cos (0.00005 f)-\frac{10345.1 \sin (0.00005 f)}{f}\right) \\
\cdot \sin (0.00006 f)]+\cos (0.000013 f) \\
\cdot[\cos (0.00006 f)(1.5 \cos (0.00005 f) \\
\left.-\frac{10345.1 \sin (0.00005 f)}{f}\right) \\
-0.0000567 i \cdot\left(-\frac{1.8252 \cdot 10^{8} i \cos (0.00005 f)}{f}\right. \\
-26464.78 i \sin (0.00005 f) \\
-\frac{1.89 \cdot 10^{12} i \sin (0.00005 f)}{f^{2}} \\
\left.+\frac{2.7378 \cdot 10^{8} i \sin (0.00005 f)}{f}\right)
\end{gathered}
$$

Higher mode frequencies of the designed transducer are calculated using Eq. (5).

The second and third mode frequencies of the transducer are estimated as $70.1 \mathrm{kHz}$ and $109.8 \mathrm{kHz}$.

\section{SIMULATION RESULTS}

Using the sizes and materials in Table 1, we perform the finite element analysis using Ansys 14.5, where the constants for the materials are borrowed from the samples provided by Ansys 14.5. We begin with the simulation of resonance frequencies of the transducer using the mode analysis. The first, second and third resonance frequencies from the analysis of the results are about $23045 \mathrm{~Hz}, 70714 \mathrm{~Hz}$, and $115436 \mathrm{~Hz}$, respectively.

Figure 2 depicts the displacement distributions of the resonance frequencies.

It can be seen that the longitudinal vibration appears in three resonance frequencies. The first and second vibrations are only 


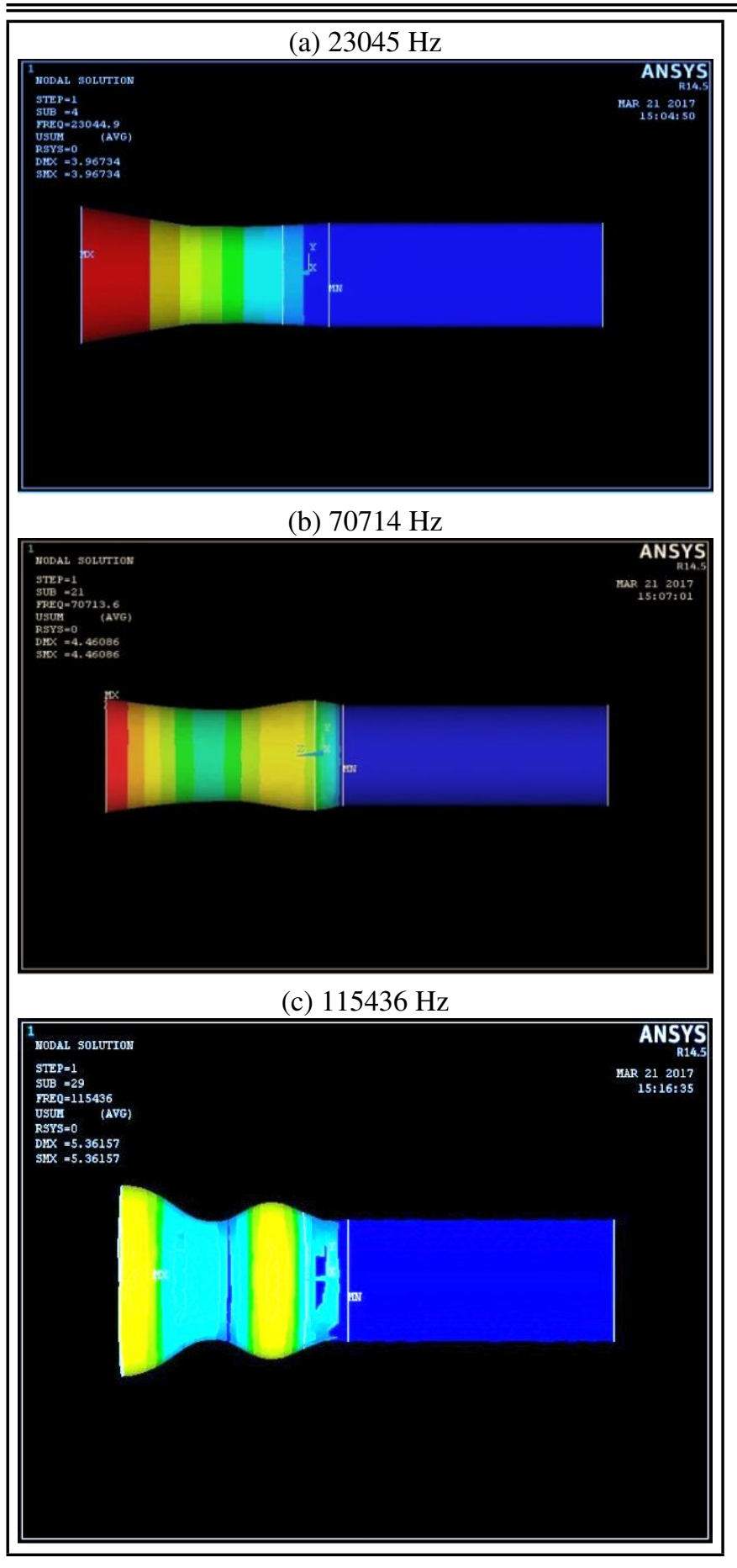

Figure 2. Displacement distributions of the transducer.

longitudinal, but the third one is a combination of the longitudinal and transversal.

Figure 2 also illustrates that the node locations of the vibrations are good with the designed ones. The differences between the theoretical and analytical results are about $3 \mathrm{kHz}$, $600 \mathrm{~Hz}$, and $5 \mathrm{kHz}$ in the first, second, and third modes.

In the analysis, the sound pressure fields of the resonance frequencies are simulated.

The sizes of the fields satisfy the far-field condition $\left(2 a_{2} / \lambda>1\right)$.

The simulation is confined to the half part from the symmetry of the system.

Figure 3 shows the sound pressure field at each resonance

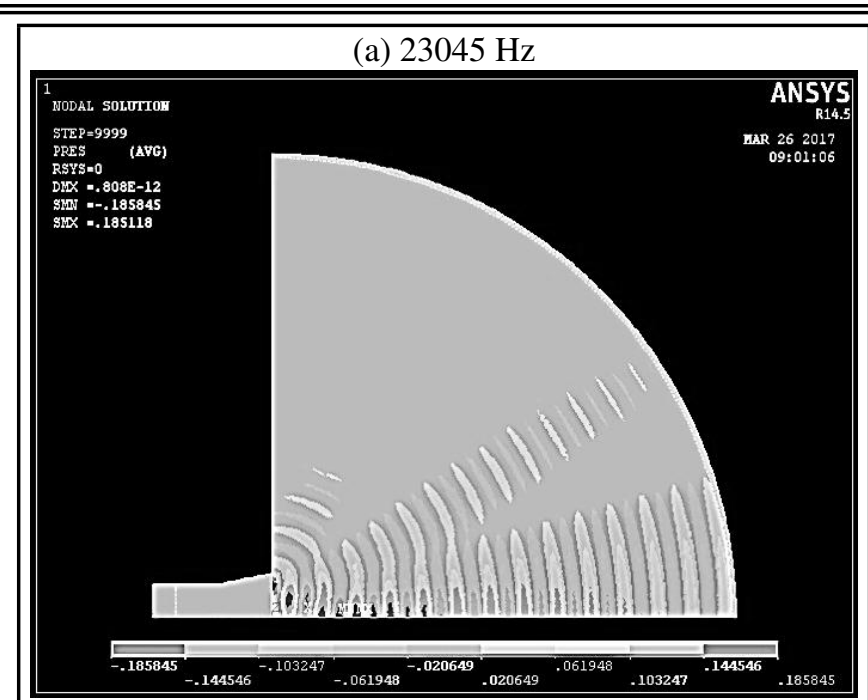

(b) $70714 \mathrm{~Hz}$

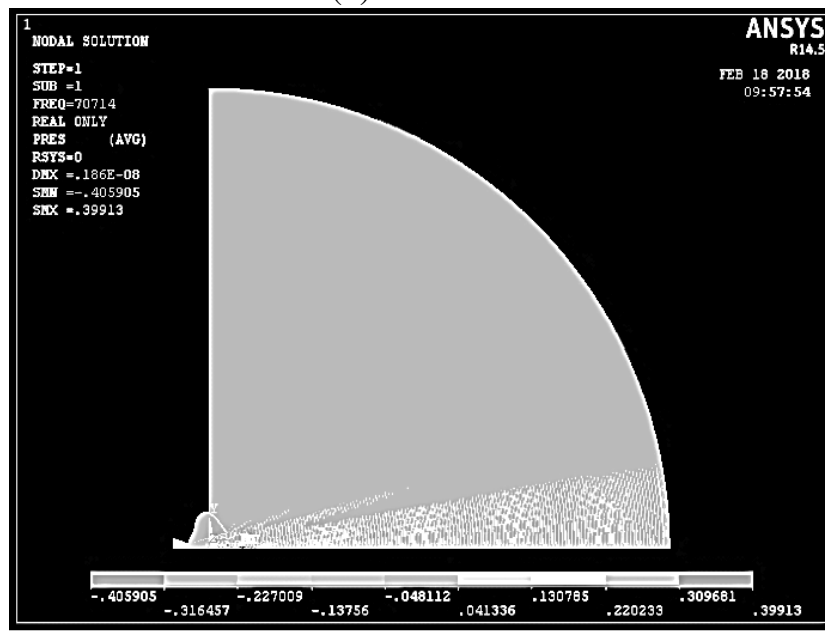

(c) $115436 \mathrm{~Hz}$

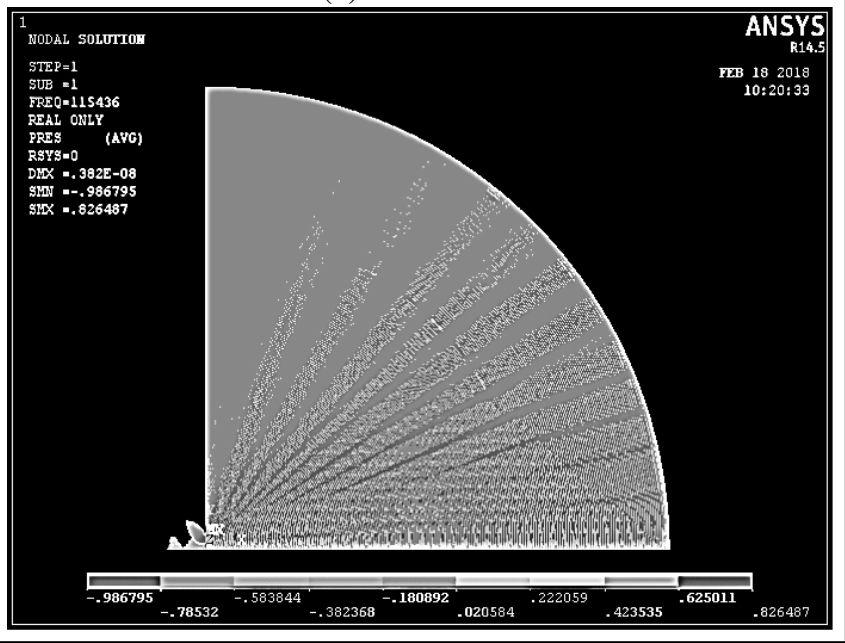

Figure 3. The sound pressure fields

frequency.

The simulation results for the sound pressure field clarify the vibrational features and of the transducer and radiation features of the sound energy at the determined frequencies. From Fig. 3a, it is obvious that most of the sound energy radiated by the transducer at the natural frequency propagates along the axis of symmetry, except some energy propagating along one or two directions off the axis. This claims that the vibration at 
the resonance frequency should be longitudinal.

The vibration at the second mode is also longitudinal with a smaller energy along the axis of symmetry but a higher directivity than the ones at the natural frequency (Fig. 3b). On one hand, at the second mode, there is no definite directional radiation off the axis of symmetry identified and the sound pressure tends to be less smooth as the angle increases, which shows that the radial vibration should be accounted even though the longitudinal one still dominates.

The sound pressure distribution at the third mode is more complicated. The sound energy diverges from the transducer and has a few peaks with decreasing intensities as the angles between the axis and the radiations increase. In particular, the dominant direction of radiation inclines $5^{\circ}$ off the axis of symmetry, and the difference between the pressures of the dominant and the remaining is comparatively smaller than the cases above. This concludes that the vibration at this frequency is the combination of the longitudinal and the radial.

In order to characterize the directivity of the transducer at each resonance frequency, the directivities of every resonance frequencies are calculated from the fields of Fig. 3.

The directional angles are about $20^{\circ}$ and $10^{\circ}$ in the first and second mode. In the third mode, the directivity is a heart shape. The cause seems to be that the third vibration is a combination of longitudinal and transversal one. In the third directivity, the angle of the right (or left) peak is about $5^{\circ}$, and the angle between the two peaks' centres is about $10^{\circ}$. All of the directivities can be used to estimate the directivity of a difference frequency when the nonlinear effect appears by the designed transducer.

\section{CONCLUSIONS}

In the paper, the transform matrix method was used to estimate the high mode frequencies of the transducer which does not satisfy the one-dimensional condition and radiates two primary waves for the nonlinear effect at the same time. The second and third mode frequencies of the transducer were estimated as $70714 \mathrm{~Hz}$ and $115436 \mathrm{~Hz}$, which were determined by the transfer matrix method for the case of the transducer with the natural frequency of $20 \mathrm{kHz}$ under the one-dimensional condition. Using the resonance frequencies of the designed transducer, the difference frequencies of about $45-47 \mathrm{kHz}$ (between the first and the second or the second and third) or $92.4 \mathrm{kHz}$ (between the first and the third) could be obtained. Also, the results of the method were compared with ones from the finite element analysis.

The higher mode frequencies of the transducer, which does not satisfy the one-dimensional condition were estimated with the difference of less than about $6 \mathrm{kHz}$ by our method. Additionally, the directivity of the sound pressure field radiated by the transducer at the determined frequencies were analysed from the simulation, which is necessary for the directivity and the frequencies of the difference frequency wave in the presence of nonlinear interactions.

When the nonlinear effect appears by the transducer designed using this method, the frequency of the difference wave and its directivity may be estimated. (a) $23045 \mathrm{~Hz}$

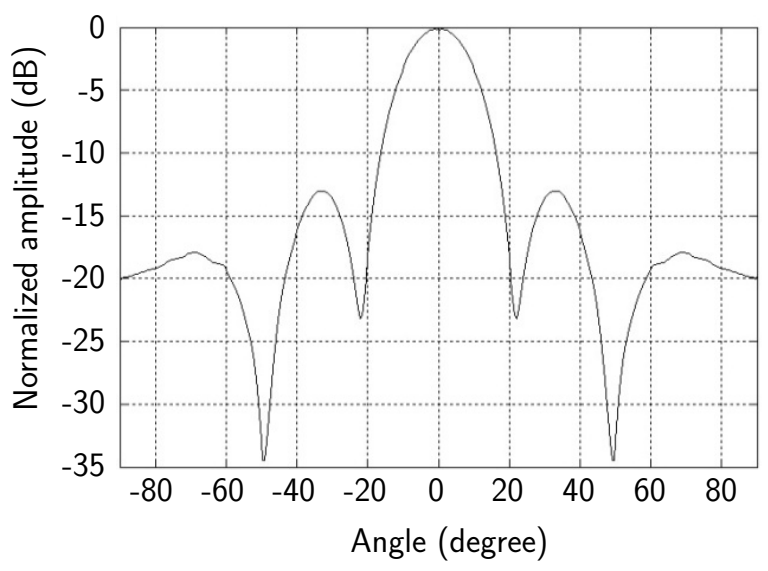

(b) $70714 \mathrm{~Hz}$

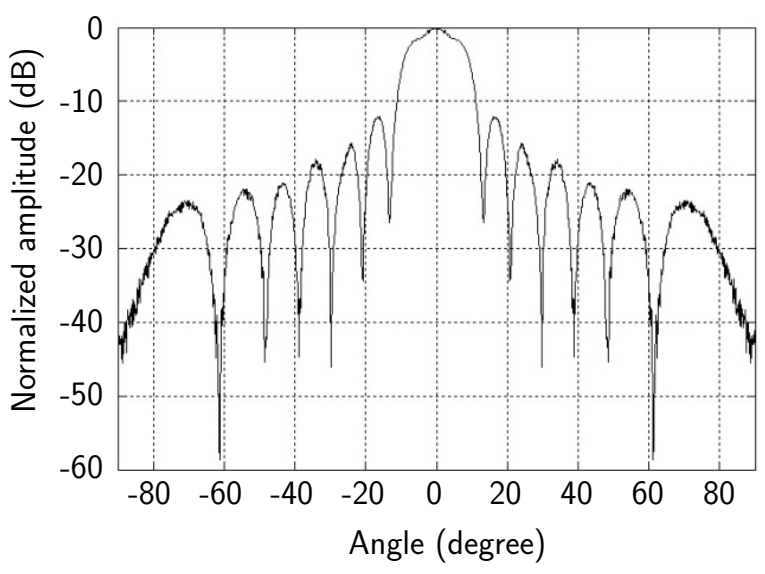

(c) $115436 \mathrm{~Hz}$

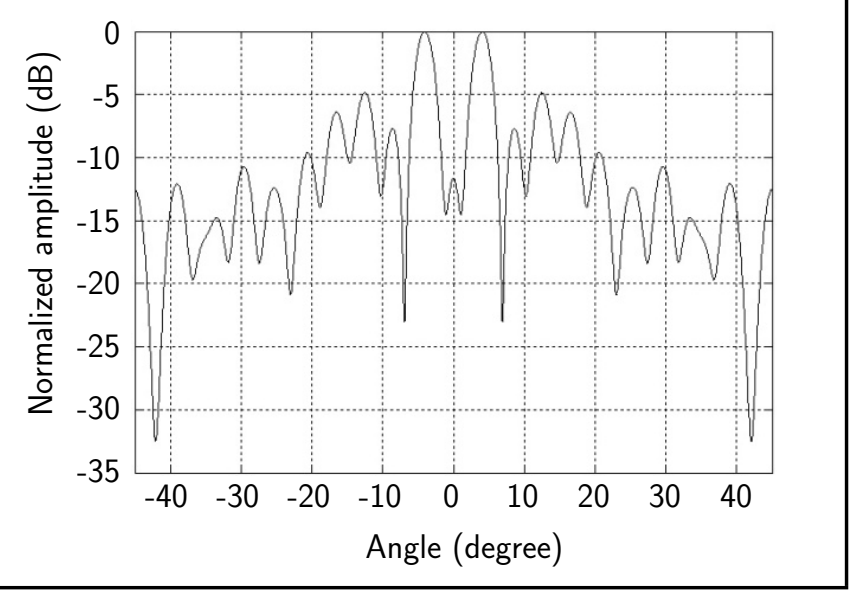

Figure 4. The directivities of the resonance frequencies calculated from the sound fields of Fig. 3.

\section{REFERENCES}

1 Webster, J. G. The Measurement, Instrumentation and Sensors Handbook, CRC Press, 1999.

2 Noykov, S. and Roumenina, C. Calibration and interface of a Polaroid ultrasonic sensor for mobile robots, Sensors and Actuators A: Physical 135 (1) 169-178, (2007). https://dx.doi.org/10.1016/j.sna.2006.07.006. 
3 http://www.engr.udayton.edu/faculty/jloomis/ece445/topics /sonar/6500.pdf

4 Armitage, P. R. and Wright, C. D. Design, development and testing of multi-functional non-linear ultrasonic instrumentation for the detection of defects and damage in CFRP materials and structures. Composites Science and Technology, 87, 149-156 (2013). https://dx.doi.org/10.1016/j.compscitech.2013.07.014.

5 Dos Santos, S., Goursolle, T., Drab, M., and Slunecko, T. Chirp-coded excitation applied with advanced pulse inversion for nonlinear acoustics in complex steel samples. The Journal of the Acoustical Society of America, 123 (5) 33993399, (2008). https://dx.doi.org/10.1121/1.2934088.

6 Chaziachmetovas, A., Svilainis, L., Kybartas, D., Aleksandrovas, A., and Liaukonis, D. Evaluation of material nonlinearities using rectangular pulse trains for excitation, Physics Procedia, 70, 582-585 (2015). https://dx.doi.org/10.1016/j.phpro.2015.08.026.

7 Goursolle, T., Dos Santos, S., Matar, O. B., and Calle, S Non-linear based time reversal acoustic applied to crack detection: Simulations and experiments. International Journal of Non-Linear Mechanics, 43 (3), 170-177, (2008). https://dx.doi.org/10.1016/j.ijnonlinmec.2007.12.008.

8 Blanloeuil, P., Meziane, A., and Bacon, C. 2D finite element modeling of the non-collinear mixing method for detection and characterization of closed cracks. NDT \& E International, 76, 43-51, (2015). https://dx.doi.org/10.1016/j.ndteint.2015.08.001.

9 Jhang, K. Y. Nonlinear ultrasonic techniques for nondestructive assessment of micro damage in material, International Journal of Precision Engineering and Manufacturing, 10 (1), 123-135, (2009). https://dx.doi.org/10.1007/s12541-017-0018-3.

10 Delrue, S., Aleshin, V., Truyaert, K., Matar, O. B., and Van Den Abeele, K. Two dimensional modeling of elastic wave propagation in solids containing cracks with rough surfaces and friction - Part II: Numerical implementation, Ultrasonics, 82, 19-30, (2018). https://dx.doi.org/10.1016/j.ultras.2017.07.003.

11 Novikov, B. K., Rudenko, O. V., and Timoshenko, V. I. Nonlinear Underwater Acoustics, Leningrad: American Institute of Physics, 1987.

12 Hunter, T. F. and Bolt, R. H. Sonics Acoustical Society of America Through the American Institute of Physics, (2000).

13 Naugolnykh, K. and Ostroysky, L. Nonlinear Wave Process in Acoustics, Cambridge University Press, 1987.

14 Bennett, M. B. and Blockstock, D. T. Parametric array in air, The Journal of the Acoustical Society of America 57 (3), 562-568, (1975). https://dx.doi.org/10.1121/1.380484.
15 Hamilton, M. F. Sound beams. Nonlinear Acoustics, San Diego: Academic, 233-261, (1998).

16 Darvennes, C. M. and Hamilton, M. F. Scattering of sound by sound from two Gausian beams, The Journal of the Acoustical Society of America 87, 1955-1964, (1990). https://dx.doi.org/10.1121/1.399322.

17 Yoneyama, M. The audio spotlight: an application of loudspeaker design, The Journal of the Acoustical Society of America 73 (5), 1532-1536, (1998). https://dx.doi.org/10.1121/1.389414.

18 Gan, W. S., Yang, J., Tan, K. S., and Er, M. H. A digital beamsteerer for difference frequency in parametric array, IEEE Transactions on Audio, Speech, and Language Processing 14 (3), 1018-1025, (2006). https://dx.doi.org/10.1109/TSA.2005.857786.

19 Tanaka, N. and Tanaka, M. Active noise control using steerable parametric array loudspeaker, The Journal of the Acoustical Society of America 127 , 3526-3537, (2011). https://dx.doi.org/10.1121/1.3409483.

20 C. Shi, W. Gan, Steerable parametric loudspeaker with preprocessing methods, Proceedings of Meetings on Acoustics, 19 (1), 1-6, (2013). https://dx.doi.org/10.1121/1.4801093.

21 Fujisawa, K. and Asada, A. Nonlinear sound propagation on acoustic phased array, Applied Acoustics 95, 57-59, (2015). https://dx.doi.org/10.1016/j.apacoust.2015.02.014.

22 Shi, C. and Gan, W. S. Product directivity models for parametric loudspeakers, The Journal of the Acoustical Society of America 131 (3), 1938-1945, (2012). https://dx.doi.org/10.1121/1.3682035.

23 Cai, C., Liu, G. R., and Lam, K. Y. A technique for modelling multiple piezoelectric layers, Smart materials and structures, 10(4), 689, (2001). https://dx.doi.org/10.1088/0964-1726/10/4/312.

24 Tiere, P. and Decarpingny, J. N. Design of high power ultrasonic transducers for use in macrosonics, Proceedings of the International Workshop on Power Sonic and Ultrasonic Transducers Design, Springer Verlag Berlin, FRG, 185-207, (1988).

25 Li, J. J., Li, Z. Y, and Zhang, D. Z. Second harmonic generation in one-dimensional nonlinear photonic crystals solved by the transfer matrix method, Physical Review E 75 (5), 056606, (2007). https://dx.doi.org/10.1103/PhysRevE.75.056606. 